$F \cdot\left(C_{1} \cdot C_{2}\right) \neq \varnothing$. Choose $p$ an element of the set $F \cdot\left(C_{1} \cdot C_{2}\right)$. Now since $F \subset C_{\beta}$ for every $\beta$, it follows that $p$ is an element of their intersection.

In the case that the union of no two sets separates the plane it follows that the intersection of any two sets is connected. The proof of the theorem is then completed by the use of Theorem 1.

\title{
REFERENCES
}

1. Josef Molnar, Über den zweidimensionalen topologischen Satz von Helly, Mat. Lapok 8 (1957), 108-114.

2. R. L. Wilder, Topology of manifolds, Amer. Math. Soc. Colloq. Publ. Vol. 32, Amer. Math. Soc., Providence, R. I., 1949.

Arkansas College and

OKLAHoma State University

\section{A NOTE ON EXACT SEQUENCES}

D. G. MALM

1. We consider a commutative diagram of abelian groups with exact rows :

$$
\begin{aligned}
& A_{1} \rightarrow A_{2} \stackrel{\beta}{\rightarrow} A_{3} \stackrel{\gamma}{\rightarrow} A_{4} \rightarrow A_{5} \\
& \downarrow \phi_{1} \quad \downarrow \phi_{2} \quad \downarrow \phi_{4} \quad \downarrow \phi_{5} \\
& B_{1} \rightarrow B_{2} \rightarrow B_{3} \rightarrow B_{4} \rightarrow B_{5}
\end{aligned}
$$

FIGURE 1

We suppose the $\phi_{i}$ are isomorphisms onto. The five lemma states that if $\phi_{3}: A_{3} \rightarrow B_{3}$ is a homomorphism for which the diagram (with $\phi_{3}$ inserted) commutes, then $\phi_{3}$ is an isomorphism onto.

Suppose $\eta_{1}, \eta_{2}: A_{3} \rightarrow B_{3}$ both give commutative diagrams, so that each is an isomorphism onto. (It is easy to find examples in which $\eta_{1} \neq \eta_{2 .}$.)

Proposition I. Under the above hypotheses,

$$
\eta_{1}^{-1} \eta_{2}(x)+\eta_{2}^{-1} \eta_{1}(x)=2 x
$$

for all $x \in A_{3}$.

To prove this, let $f=\eta_{1}^{-1} \eta_{2}$, and let $I$ be the identity function on $A_{\mathbf{3}}$.

Received by the editors March 13, 1962. 
The equation reads $f+f^{-1}=2 I$, which is easily seen to be equivalent to $(f-I)(f-I)=0$. In fact, applying $f$ to $f+f^{-1}=2 I$, we get $(f-I)^{2}$ $=0$. In proving the reverse implication, the only question is whether $f$ has an inverse. However, $f$ is onto, for if $y \in A_{3}$, then $f(y-(f-I) y)$ $=y$. Also $f$ is $1-1$, for if $f(x)=0$, then $(f-I)(x)=-x$, and applying $(f-I)$ again, we see that $0=(f-I)(-x)=x$. To see that $(f-I)^{2}=0$, we observe that $(f-I) \beta=0$ and that $\gamma(f-I)=0$. Thus image $(f-I)$ Ckernel $\gamma=$ image $\beta$, and $(f-I)^{2}=0$.

Note that the extremities of the diagram were not used in the proof, except to insure that $\eta_{1}$ and $\eta_{2}$ were isomorphisms onto. In fact, we may reason to the same conclusion from the commutative diagram

$$
\begin{aligned}
& A_{2} \stackrel{\beta}{\rightarrow} A_{3} \stackrel{\gamma}{\rightarrow} A_{4} \\
& \downarrow \quad \eta_{1} \downarrow \downarrow \eta_{2} \quad \downarrow \phi_{4} \\
& B_{2} \rightarrow B_{3} \rightarrow B_{4}
\end{aligned}
$$

where $\eta_{1}$ and $\eta_{2}$ are isomorphisms onto, $\phi_{4}$ is $1-1$, and the rows are exact.

Now suppose (in either diagram) that $A_{3}$ and $B_{3}$ are rings, and $\eta_{1}: A_{3} \rightarrow B_{3}$ is a ring isomorphism onto making the diagram commute. We consider the question of whether there is another ring isomorphism $\eta_{2}: A_{3} \rightarrow B_{3}$ making the diagram commute.

Proposition II. A necessary and sufficient condition for the existence of a ring isomorphism $\eta_{2}: A_{3} \rightarrow B_{3}$ making the diagram commute, with $\eta_{2} \neq \eta_{1}$, is that there exist a nontrivial additive homomorphism $\delta: A_{3} \rightarrow A_{3}$ such that

(i) $\delta \beta=0, \gamma \delta=0$ (consequently, $\delta^{2}=0$ ) and

(ii) $\delta(x y)=(\delta x) y+x \delta y+(\delta x)(\delta y)$ for all $x, y \in A_{3}$.

PRoof. If such a $\delta$ exists, define $\eta_{2}=\eta_{1}+\eta_{1} \delta$. It is trivial that $\eta_{2}$ is a ring homomorphism, that the diagram commutes, and that $\eta_{2}$ is 1-1. To verify that $\eta_{2}$ is onto, let $y \in B_{3}$, and let $\eta_{1}(x)=y$. Then $\eta_{2}(x-\delta x)=y$. On the other hand, suppose $\eta_{1}$ and $\eta_{2} \neq \eta_{1}$ are given. Let $\delta=\eta_{1}^{-1} \eta_{2}-I$. Then (i) and (ii) are easily verified.

REMARKs. Applying $\delta$ to (ii), we get

$$
2(\delta x)(\delta y)=0 \quad \text { for all } x, y \in A_{3} .
$$

Since image $\delta \subset$ image $\beta$, in order for $\delta$ to be nontrivial it is necessary that image $\beta$ contain divisors of zero or elements of order two.

If $A_{3}$ has a two-sided identity element 1 , then it follows that $\delta(1)$ $=0$. 
An example in which $\eta_{1} \neq \eta_{2}$ is given by Figure 1 , where the two rows are identical, $A_{1}=A_{5}=\{0\}, A_{2}=A_{4}=Z_{2}$ (the integers modulo two), $A_{3}=B_{3}=Z_{2} \times Z_{2}, \phi_{i}=$ identity $=\eta_{1}, \eta_{2}(x, y)=(y, x), \beta(x)=(x, x)$, and $\gamma(x, y)=x+y$, for $x, y \in Z_{2} . \eta_{1}$ and $\eta_{2}$ are both ring isomorphisms.

2. An application. We consider $(k-1)$-sphere bundles $B^{\prime}=\left(E^{\prime}, p^{\prime}, B^{\prime}, S^{k-1}, S O(k)\right)$ and $B=\left(E, p, B, S^{k-1}, S O(k)\right)$ with group the special orthogonal group $S O(k)$; and two bundle maps $f, g: \mathbb{B}^{\prime} \rightarrow \mathbb{B}$. Let $\bar{f}, \bar{g}$ be the mappings of the base spaces induced by $f$ and $g$, respectively. We use a field $F$ of characteristic not two for coefficients for cohomology, and we assume that $\bar{g}^{*}=\bar{f}^{*}: H^{*}(B, F)$ $\rightarrow H^{*}\left(B^{\prime}, F\right)$ and that this is an isomorphism onto. Then if $H^{*}(E, F)$ has no divisors of zero, $f^{*}=g^{*}: H^{*}(E, F) \rightarrow H^{*}\left(E^{\prime}, F\right)$. To see this, apply the remark following Proposition II to the isomorphisms of the Gysin sequences induced by $f$ and $g$ :

$$
\begin{aligned}
& \cdots \longrightarrow H^{q-k}(B) \longrightarrow H^{q}(B) \longrightarrow H^{q}(E) \longrightarrow H^{q-k+1}(B) \longrightarrow \cdots \\
& \bar{f}^{*}=\bar{g}^{*} \downarrow \quad \bar{f}^{*}=\bar{g}^{*} \downarrow \quad f^{*} \downarrow \downarrow g^{*} \quad \downarrow \bar{f}^{*}=\bar{g}^{*} \\
& \cdots \longrightarrow H^{q-k}\left(B^{\prime}\right) \longrightarrow H^{q}\left(B^{\prime}\right) \longrightarrow H^{q}\left(E^{\prime}\right) \longrightarrow H^{q-k+1}\left(B^{\prime}\right) \longrightarrow \cdots
\end{aligned}
$$

An example in which $H^{*}(E, F)$ has no divisors of zero is furnished by taking $B$ to be the universal $(k-1)$-sphere bundle $\left(B_{k-1}, p, B_{k}\right.$, $S^{k-1}, S O(k)$ ), where $B_{n}$ is the classifying space for $S O(n)$. For the cohomology of $B_{n}$ see A. Borel, Topology of Lie groups and characteristic classes, Bull. Amer. Math. Soc. 61 (1955), 397-432.

State University of New York 